\title{
Forces on oscillating uniform and tapered cylinders in crossflow
}

\author{
By F. S. HOVER, A. H. TECHET \\ AND M. S. TRIANT AF Y LLOU \\ Department of Ocean Engineering, Massachusetts Institute of Technology, \\ Cambridge, MA 02139, USA
}

(Received 19 June 1997 and in revised form 18 December 1997)

Forces are measured at both ends of rigid cylinders with span $60 \mathrm{~cm}$, performing transverse oscillations within an oncoming stream of water, at Reynolds number $R e \approx 3800$. Forced harmonic motions and free vibrations of uniform and tapered cylinders are studied. To study free motions, a novel force-feedback control system has been developed, consisting of: $(a)$ a force transducer, which measures forces on a section of a cylinder moving forward at constant speed; $(b)$ a computer using the measured force signal to drive in real time a numerical simulation of an equivalent mass-dashpot-spring system; (c) a servomotor and linear table which impose, also in real time, the numerically calculated motion on the cylinder section. The apparatus allows very low equivalent system damping and strict control of the parametric values and structure of the equivalent system.

Calculation of the cross-correlation coefficient between forces at the two ends of the uniform cylinder reveals five distinct regimes as a function of the nominal reduced velocity $V_{r n}$ : two regimes, for low and high values of $V_{r n}$, and far away from the value of $V_{r S}$ corresponding to the Strouhal frequency, show small correlation; two regimes immediately adjacent to, but excluding, $V_{r S}$ show strong correlation, close to 1 ; surprisingly, there is a regime containing the Strouhal frequency, within which correlation is low. Free vibrations with a 40:1 tapered cylinder show that the regime of low correlation, containing the Strouhal frequency, stretches to higher reduced velocities, while lock-in starts at lower reduced velocities.

When comparing the amplitude and phase of the lift coefficient measured for free and then for forced vibrations, we obtain close agreement, both for tapered and uniform cylinders. When comparing the cross-correlation coefficient, however, we find that it is much higher in the forced oscillations, especially for the uniform cylinder. Hence, although the force magnitude and phase may be replicated well in forced vibrations, the correlation data suggest that differences exist between free and forced vibration cases.

\section{Introduction}

The interaction of a slender cylindrical body with a steady crossflow has been the topic of a great amount of attention, because of the complexity of the viscous flow problem, particularly when interacting dynamically with a structure. The problem is significant both as a paradigm for studying bluff body-flow interaction, and as a practical issue. Engineering applications include aeolian vibrations of transmission lines, and marine risers and cables strumming within a current. For a wide range 
of physical parameters, vortex-induced vibration (VIV) leads to spatially extended motions of the structure, often resulting in large dynamic stresses.

The prediction of VIV is currently based on semi-empirical methods, often employing a 'strip theory' approach and relying on experimental data bases, which are obtained through a number of simplifying assumptions (Blevins 1990; Naudascher \& Rockwell 1994). Major outstanding problems include the correlation length effects, particularly in non-uniform cylinders and sheared oncoming flows, and the effect of multi-frequency response. Parallel experimental and numerical efforts are underway to provide better understanding of the phenomenon and develop reliable methods of prediction. Coutanceau \& Defaye (1991) and Williamson (1996) provide recent reviews of progress made in understanding the basic mechanisms of flow response.

The need to generate a complete experimental force data base for predicting vortexinduced vibrations of slender structures has led to testing of cylinders undergoing forced oscillations. Several investigators, including Bishop \& Hassan (1964), Sarpkaya (1977), Staubli (1983), Gopalkrishnan (1992) and Gopalkrishnan, Grosenbaugh \& Triantafyllou (1992) have measured the forces on finite-span oscillating cylinders forced in harmonic as well as multi-frequency motion. In harmonic tests the transverse (lift) force is decomposed into two components: one in phase with velocity and the other in phase with acceleration. The former is important in determining the range where self-excited oscillations occur; the second is used to derive a value for the added mass coefficient.

Predictions using forced-vibration data with a structural model have been compared with free vibration tests (Staubli 1983; Parkinson 1989; Sarpkaya 1995): there are parametric regions where such comparison is successful, and other regions where discrepancies are observed. The question of whether forced cylinder experiments can be used to predict free vibrations of cylinders is still open. For example, Griffin (1972) compared the wakes of two identical cylinders, one self-excited and the other forced to oscillate. He did not find differences in the global parameters of the wake, although he noted differences in the velocity signal of the free-oscillation wake, which contained more random oscillations. Newman \& Karniadakis (1996) find through numerical simulation differences in the wake structure of a cable forced to oscillate compared with the wake of a self-excited cable. The explanation may be based on the dynamics of the wake; for example, it is known (Nakano \& Rockwell 1994) that amplitude modulation is capable of altering the wake form. A freely vibrating cylinder can have a different wake structure (and force signal) since it can deviate from a purely harmonic motion.

In this paper, we consider forced as well as free vibrations to study the effect of the principal parameters on the integrated force acting on a finite span cylinder, at Reynolds number 3800 . A hybrid system is developed to study free motions, employing a closed-loop control system consisting of: a pair of force transducers measuring the transverse forces at both ends of a test cylinder moving forward at constant speed; a dedicated computer which uses in real-time the measured force to drive a numerical simulation of an equivalent mass-dashpot-spring system; a servomotor and linear table which impose, also in real-time, the numerically calculated motion to the cylinder section. The apparatus results in an effective mass-dashpot-spring system vibrating freely within crossflow, and allows very low effective damping.

We present new data in agreement with those of Feng (1968), Brika \& Laneville (1993), and Khalak \& Williamson (1996). In addition, we use the two simultaneously recorded end forces directly, to assess spanwise correlation of the fluid structure for the four cases of: (a) a uniform cylinder in free vibration, $(b)$ a 40:1 tapered cylinder 


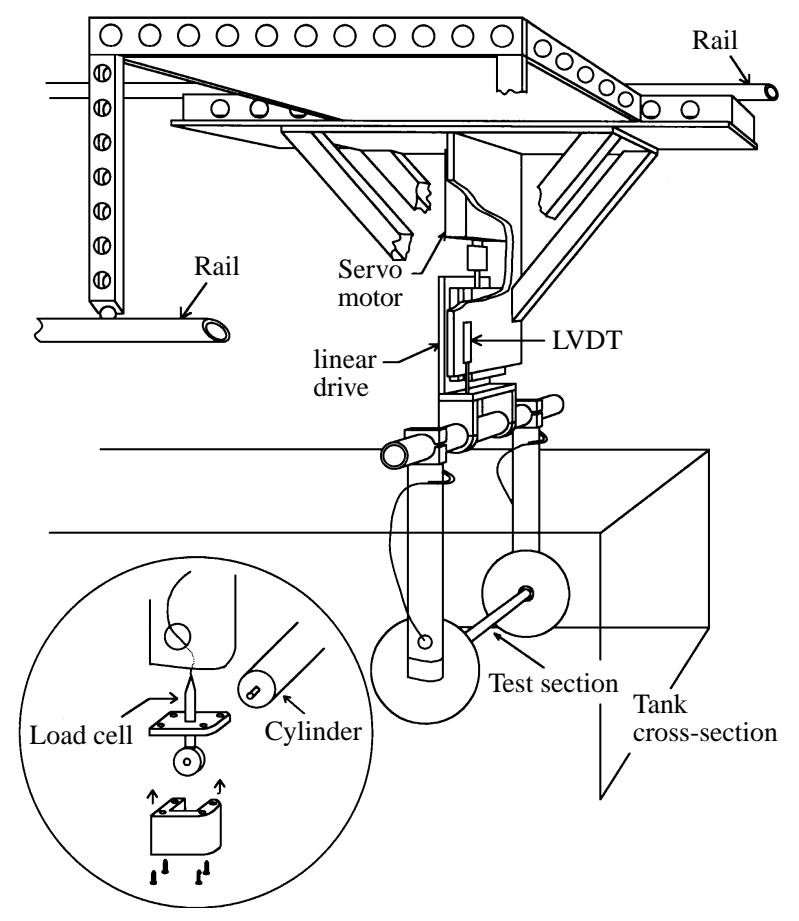

FIGURE 1. Test section and carriage apparatus.

in free vibration, (c) a uniform cylinder in forced vibration, and $(d)$ a 40:1 tapered cylinder in forced vibration. Through the force feedback mechanism, we are able to compare force responses in forced sinusoidal motions and free vibrations with the same apparatus and under identical flow conditions.

\section{Description of experimental apparatus}

The experiments were conducted at the MIT Testing Tank Facility. The main stillwater tank has dimensions $30 \mathrm{~m} \times 2.5 \mathrm{~m} \times 1.2 \mathrm{~m}$. As shown in figure 1 , the tank is equipped with a double-rail structure supporting a motor-driven carriage, which can achieve speeds from 0.1 to $1.5 \mathrm{~m} \mathrm{~s}^{-1}$. The test section of the cylinder is $62 \mathrm{~cm}$ long and fits into an inverted U-frame yoke assembly which hangs down from the carriage into the water. Sensitive load cells form the junction between the cylinder and the yoke at both ends; the ends are fitted with two circular endplates of $35 \mathrm{~cm}$ diameter to ensure two-dimensional conditions and to avoid end-effects. The diameter of the uniform cylinder is $3.17 \mathrm{~cm}$, resulting in an aspect ratio of 19.2. The tapered cylinder has the same length, but a mean diameter of $2.78 \mathrm{~cm}$. During tests, the cylinder was submerged at its average position $0.60 \mathrm{~m}$ below the free surface, to avoid free surface and bottom interference effects.

The yoke and cylinder are affixed to an ALM linear lead-screw table, driven by a Parker-Hannifin servomotor and MEI motor controller card, which provide the means for oscillating the yoke with amplitude up to $8 \mathrm{~cm}$. An on-board computer provides trajectories for the servomotor, based on either forced sinusoids or a compliance model. As shown in figure 2, for the latter case we drive an on-line simulation of a compliant system with the force signals as follows: $M \ddot{x}+B \dot{x}+K x=F_{\text {hydrodynamic }}$; $s$ denotes the Laplace transform variable. It is important to note that the actual 


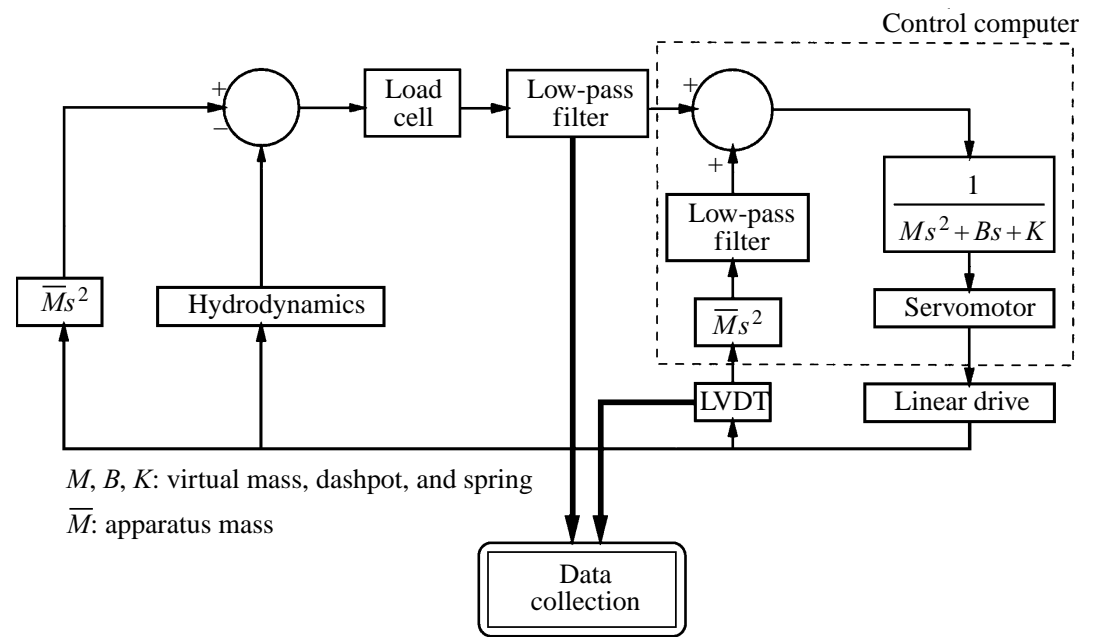

FIGURE 2. Force-feedback control system for free vibrations.

measured force comprises both the hydrodynamic force as well as one resulting from the inertia of the test cylinder and its fittings. Therefore, to obtain the hydrodynamic force in real time, we subtract from the force signal the calculated inertia force. In this way, a wide variety of virtual masses (as well as damping and stiffness values) can be accommodated with the same test cylinder. The uniform test cylinder has a specific mass of 0.372 , enabling the virtual specific mass, $m^{*}=4 M / \pi d^{2} l$, to be well below 1. The tapered test cylinder has specific mass 1.33 .

Although the simulation can specify in principle zero damping, in practice this is difficult to achieve reliably. The use of a servomotor and feedback loop carries no guarantee of zero net power flow when the simulation damping is zero, and small timing errors can push the closed-loop system into an unstable oscillatory mode. The lowest consistent damping ratio we have measured in air is approximately 0.013 . The minimum mass-damping parameter $m^{*} \zeta$ for this system is thus expected to be around 0.013 (with $m^{*}=1.0$ ), and about 0.040 for the present tests. Our apparatus achieves a mass-damping ratio, $S_{g}=8 \pi^{2} S^{2} M \zeta / \rho l d^{2}$, of about 0.029 , which is comparable to that given by Khalak \& Williamson (1996), and among the lowest reported. Our apparatus allows us to carry identical end plates and is fully immersed, while it also allows testing of different equivalent systems, which may include, for example, nonlinear terms.

The forces are measured with two Kistler piezoelectric transducers, placed at both ends of the test cylinder. The tests we describe are at $R e=3800$, and hence the measured forces are quite small. The resolution of these sensors is approximately $0.005 \mathrm{~N}$, and they require real-time filtering because of the feedback system. We use an analogue fourth-order Butterworth low-pass filter, with a cutoff at $22 \mathrm{~Hz}$; a digital filter applies the same phase to the inertial correction, for consistency. This filtering imposes a phase loss of about five degrees at the nominal rate of $4.9 \mathrm{rad}$ $\mathrm{s}^{-1}$. The motor servo attains a phase loss of less than one degree over the range of frequencies encountered, and the linear drive has a manufacturer's specification of five micrometers backlash. Acoustic Doppler velocimetry tests in the tank showed that the steady forward carriage speed has fluctuations below $5 \%$ at speeds near 12 $\mathrm{cm} \mathrm{s}^{-1}$.

A Schaevitz Linear Variable Differential Transformer (LVDT) HR 3000 with a 
linear range of $\pm 8 \mathrm{~cm}$ is used to measure the imposed motion. The Kistler transducers and the Schaevitz LVDT are operated through amplifiers located on the carriage so as to be very close to the sensors. The high-level analogue voltage outputs are sent to both the simulation/motion-control computer on the carriage, and a separate data-collection computer located away from the tank. The hardware has also been used in Hover, Miller \& Triantafyllou (1997).

In processing the data, we break up the records into bins of one to two oscillations in length, for computing the inner products listed below. We thus obtain mean values as well as sample standard deviations (RMS value); a high standard deviation indicates fluctuation over the course of the run, whereas a low value suggests that the quantity is stable. The following parameters are used to describe our results:

(a) The nominal reduced velocity, based on the structural vibration frequency: $V_{r n}=U /\left(f_{n} d\right)$.

(b) The true reduced velocity, based on the observed frequency of vibration: $V_{r}=$ $U /\left(f_{o} d\right)$.

(c) The lift-coefficient in phase with velocity, defined as

$$
C_{l v}=\frac{F_{l}}{\frac{1}{2} \rho d L U^{2}},
$$

where $F_{l}$ is the lift force in phase with velocity, computed as an inner product of (inertia-corrected) force with velocity. For the tapered cylinder, we base lift coefficients on the local diameter at each end.

(d) The lift coefficient in phase with acceleration, $C_{l a}$, defined similarly. The added mass is positive if $C_{l a}$ is negative.

(e) The phase by which the force leads the position, $\phi$.

$(f)$ The cross-correlation between forces measured at the two ends of the test cylinder, $F_{c}$. The sample standard deviation of this correlation coefficient, based on at least sixty bins, we denote as $\mu$. A correlation coefficient near 1 suggests uniform conditions in the primary vortical patterns of the wake across the span of the cylinder.

Tests were repeated in duplicate or triplicate.

\section{Free vibrations of a uniform cylinder}

Free-vibration tests were conducted at nominal reduced velocities $V_{r n}$ between 1 and 40 , although we concentrate primarily on results for $V_{r n}$ less than $10 . \dagger$ In this regime, our apparatus produces an amplitude curve that matches Khalak \& Williamson's (1996) curve (for $m^{*} \zeta=0.013$ ) very closely.

Figure 3 is a composite figure showing as functions of $V_{r n}:(a)$ the average onetenth highest amplitude of response, $(b)$ the force correlation coefficient and standard deviation, $(c)$ the phase between force and motion, $(d)$ the lift coefficient in phase with acceleration, $(e)$ the lift coefficient in phase with velocity, and $(f)$ the ratio of frequency of oscillation to natural frequency. Since two force signals are measured, one from each end of the cylinder section, plots $(c-f)$ show two points for each value of $V_{r n}$. The two data points are in most cases quite close to each other, often indistinguishable, but there are notable exceptions. The sum of the two lift coefficients in phase with velocity, pertaining to power flow, must be near zero for free vibrations,

$\dagger$ High nominal reduced velocities correspond to an extraordinarily high compliance: on the order of $1 \mathrm{~m} \mathrm{~N}^{-1}$ in the present system. Due to the high compliance of very long cables, testing in this regime may be valuable. 


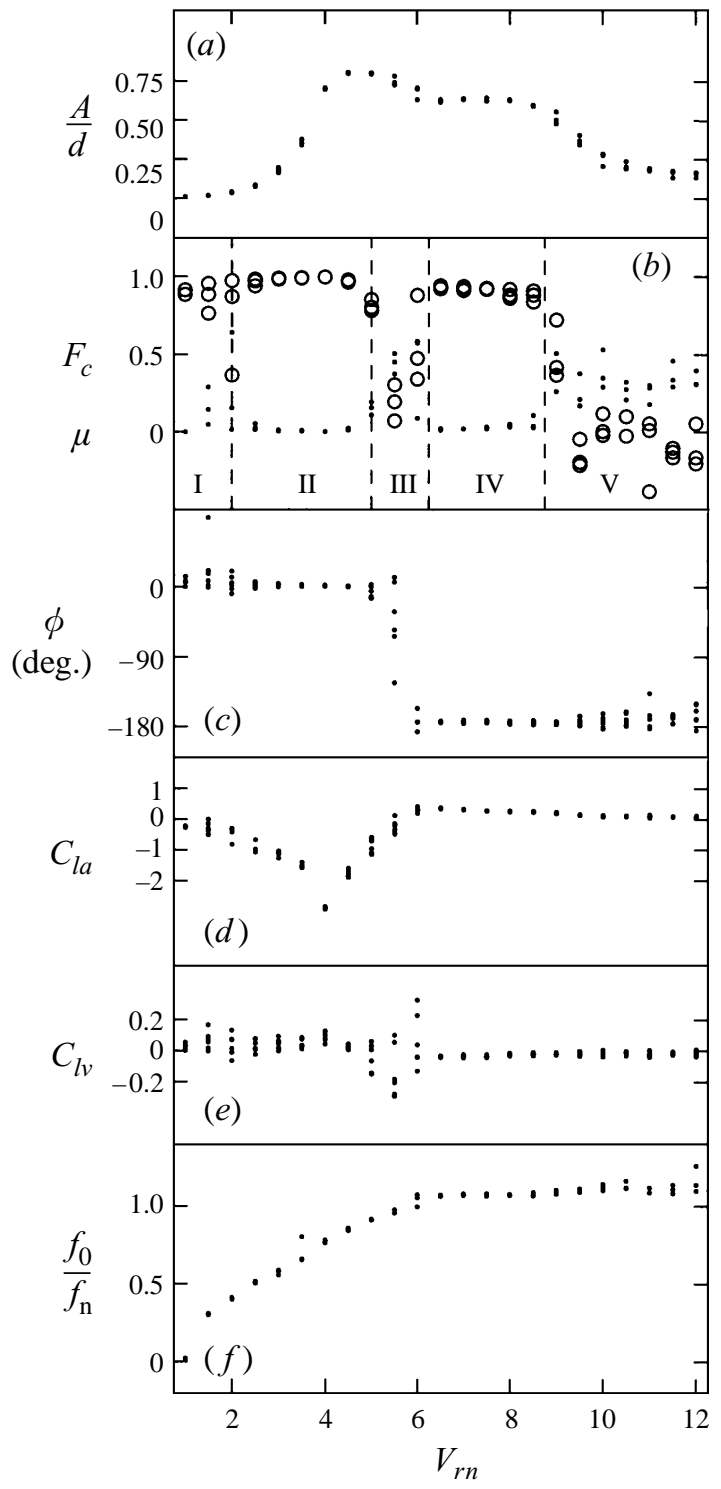

FIGURE 3. Response of a freely vibrating uniform cylinder as a function of reduced velocity: (a) amplitude to diameter ratio, $A / d$; $(b)$ correlation coefficient $F_{c}$ and standard deviation $\mu$ between forces measured at the ends; $(c)$ phase angle $\phi$ (degrees) between force and displacement; $(d)$ lift coefficient in phase with acceleration $C_{l a} ;(e)$ lift coefficient in phase with velocity $C_{l v} ;(f)$ frequency of oscillation over natural frequency $f_{o} / f_{n}$.

since the structural damping is small. This constraint is generally met; however, the individual lift coefficients are not zero in certain regimes, particularly at reduced velocity values in the range $5<V_{r n}<6$. This range contains the Strouhal frequency, and hence is very important for applications.

A sharp phase change is noted in figure $3(c)$, characteristic of the transition from the upper to the lower hysteretic branch. The lift coefficient in phase with acceleration, which has the opposite sign to the added mass force, is negative at low reduced velocities, reaching a minimum value of nearly -3.0 at $V_{r n}=4.0$, where 


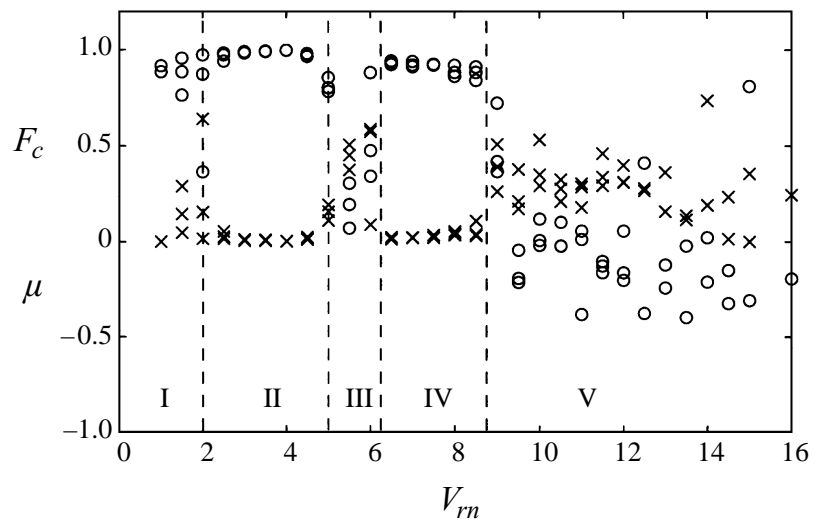

FIGURE 4. Force correlation coefficient $F_{c}(\circ)$ and standard deviation $\mu(\times)$, for free-vibration tests with the uniform cylinder.

extreme sensitivity to small variations in $V_{r n}$ is noted. Beyond this reduced velocity, $C_{l a}$ becomes smaller, first reaching a local maximum of about 0.4 , and eventually tapering off to zero.

There is a direct relation between variations in amplitude, lift, and phase, and the calculated correlation coefficients of the measured forces at the two ends of the cylinder. We have identified five different regimes as a function of $V_{r n}$, labelled I-V, and shown in figure 4, which is an expanded version of figure $3(b)$, providing finer detail. The end forces show excellent correlation in the ranges: II for $V_{r n}=2.0-5.0$, and IV for $V_{r n}=6.25-8.75$. Region II corresponds to a well-correlated ' $2 \mathrm{~S}$ ' response, while region IV is a correlated '2P' regime (Williamson \& Roshko 1988). At the lower reduced velocities (region I) the correlation coefficient is close to a value of 0.85 , while for high $V_{r n}$ values (region $\mathrm{V}$ ) the correlation is typically negative, with increased scatter.

Whereas it is anticipated that the correlation will be poor in regions I and V, far away from the Strouhal frequency, it is surprising to find poor correlation also in region III, for $V_{r n}=5.0-6.25$, which contains the Strouhal frequency. The correlation dips to a value of zero, with a corresponding increase in the value of the standard deviation $\mu$.

In figure 5, we show amplitude and force power spectra at values of $V_{r n}$ corresponding to the lower and upper limits of each of the five regions. Region I contains forcing at the Strouhal shedding rate, and a second harmonic is just visible, growing with $V_{r n}$. The transition from region I to II results in a sharpening of the displacement peak, and a shift in the higher harmonics of the forces. The lift in phase with acceleration reaches its extreme value in the middle of the coherent ' $2 S$ ' region (II). The transition from region II to III appears through a very mild amplitude drop, consistent with a hysteretic jump occurring there; but otherwise has no visible effect on the the spectra. Synchronization of the observed frequency to the structural mode occurs at $V_{r n}=6.0$, and across region III the peaks broaden mildly. Region IV is accompanied by a renewed narrowing of the force spectra, which degenerates, however, as the fixed-cylinder shedding frequency reappears in region V. The displacement decays into a slow, broadband meander, consistent with very small restoring forces.

In the correlated regions II and IV, a strong similarity between the force signals from the two ends of the cylinder exists. Elsewhere, differences can be observed. Figure 6 demonstrates a good correlation of modulated end-forces at $V_{r n}=4.0$; in 

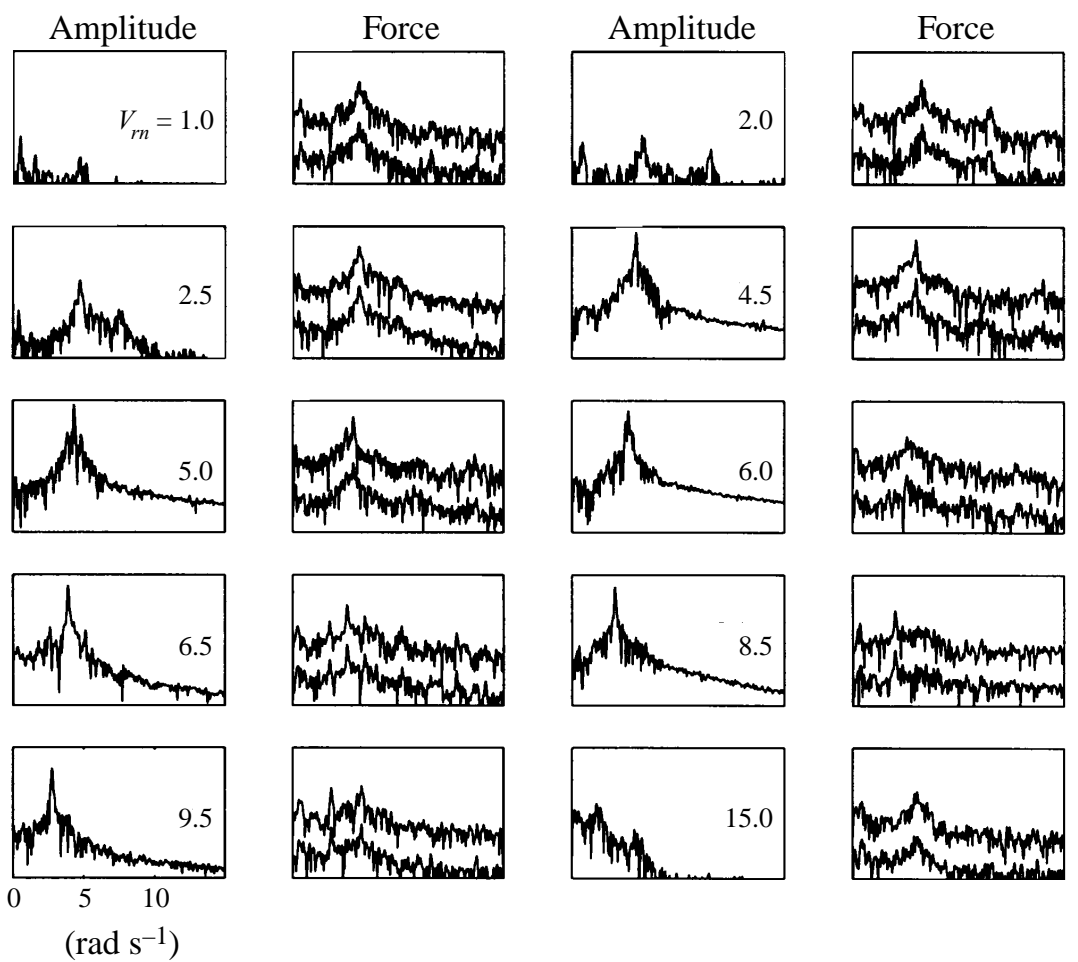

Figure 5. Amplitude and force power spectra of the uniform cylinder, at boundaries of the five regions shown in figure 4.

this case the force modulation follows the displacement modulation. On the other hand, figure 7 shows a different situation at $V_{r n}=5.5$, within region III: the two force signals are different in shape and phase, and have little apparent relation to the large, narrow-band displacement.

Figure 4 is a central result of the present investigation, showing that the forcing along the uniform cylinder section is not correlated for free vibrations close to the Strouhal frequency.

\section{Free vibrations of a 40:1 tapered cylinder}

We conducted tests for similar parametric values with a 40:1 tapered cylinder, having mass ratio 4.0. The ratio $\tau=40: 1$ represents the ratio of the cylinder length $l$ to the difference in diameters between the two ends, $d_{\max }, d_{\min }$, i.e.

$$
\tau=\left|\frac{l}{d_{\max }-d_{\min }}\right| .
$$

Figure 8 is a composite figure for the tapered cylinder results, similar to figure 3 for a uniform cylinder. As shown in figure 8 , broad variations from the uniform-cylinder results exist. In general, an averaging with respect to reduced velocity seems to occur, as also noted in Humphries (1987). The hysteretic jump near $V_{r}=6.0$ has apparently vanished and the force correlation varies more gradually.

The force correlation results for the tapered cylinder are shown in more detail in figure 9, where the low-correlation regime has shifted to the range in $V_{r n}$ between 6 
$(\mathrm{cm})$

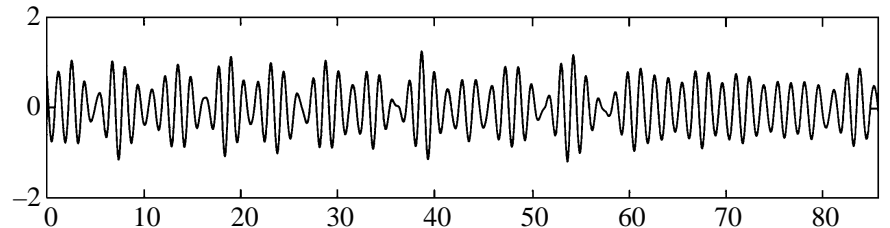

$(\mathrm{N})$

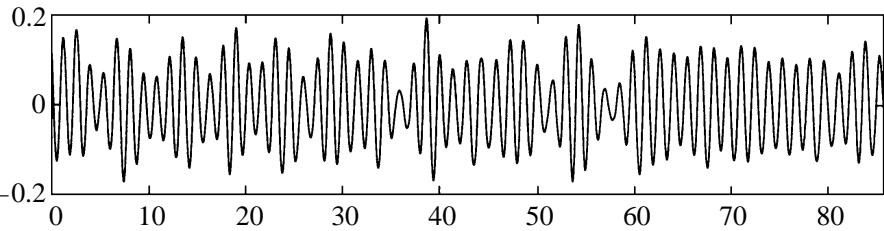

(N)

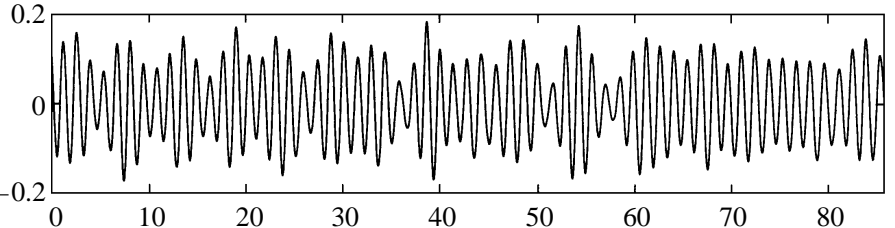

(s)

FigURE 6. Displacement and force signals from each end of the cylinder obtained for $V_{r n}=4.0$ (region II).

$(\mathrm{cm})$

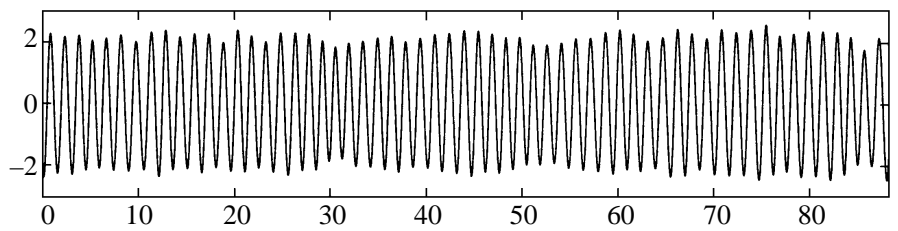

$(\mathrm{N})$

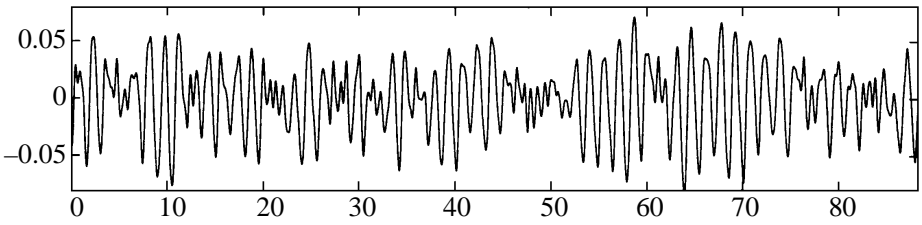

$(\mathrm{N})$

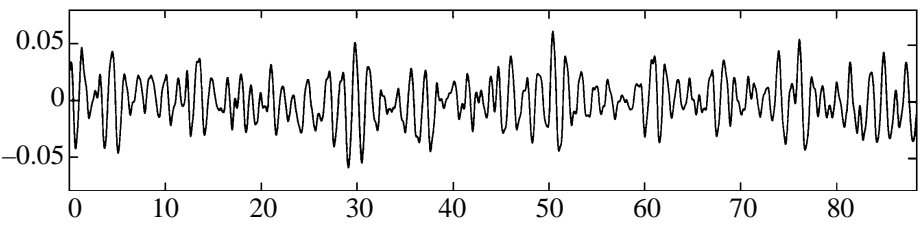

(s)

FIGURE 7. Displacement and force signals from each end of the cylinder at $V_{r n}=5.5$ (region III).

and 9, with a gradual transition. Uniform-cylinder force correlations, by comparison, appear to be alternately well-correlated or poorly correlated, with almost discontinuous transitions. The lowest correlation noted for a tapered cylinder is 0.25 , compared with 0 for a uniform cylinder.

In the plots of figure 8 force normalizations are with respect to the two end diameters of the section, so that nearly zero power flow (sum of the two end forces) again holds, when each coefficient is multiplied by its appropriate diameter. The lift 


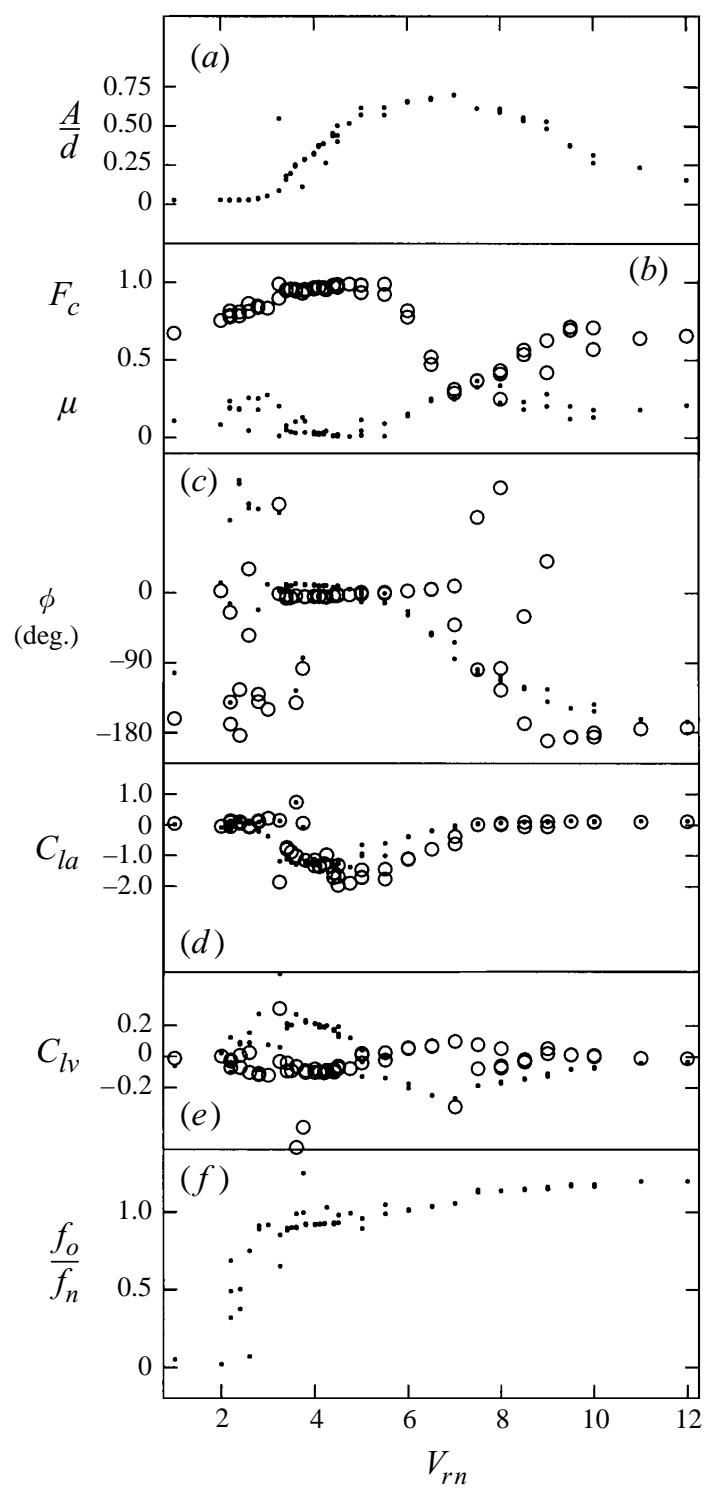

FIGURE 8 . The tapered cylinder free response characteristics: $(a)$ amplitude to diameter ratio, $A / d$; (b) correlation coefficient $F_{c}$ and standard deviation $\mu$ between forces measured at the ends; $(c)$ phase angle $\phi$ (degrees) between force and displacement; $(d)$ lift coefficient in phase with acceleration $C_{l a}$; (e) lift coefficient in phase with velocity $C_{l v} ;(f)$ frequency of oscillation over natural frequency $f_{o} / f_{n}$. For $\phi, C_{l a}$, and $C_{l v}$, and o denote the narrow and wide ends of the cylinder, respectively.

coefficients show clear separation between forces acting at the narrow end (small diameter) and the wide end (large diameter) of the test cylinder. Lift in phase with acceleration at the narrow end follows a path similar to the uniform case, with the exception that the minimum value is only -1.3 (compared with -3.0 ). At the wide end, a minimum value of -1.9 is observed at $V_{r n}=4.5$. At $V_{r n}=3.5$, a discontinuity in $C_{l a}$ is evident, although some scatter in the data exists below $V_{r n}=4.0$.

The tapered cylinder does not follow the synchronization curve of the uniform cylinder. Below $V_{r n}=3.0$, an extremely high sensitivity to the structural mode exists; 


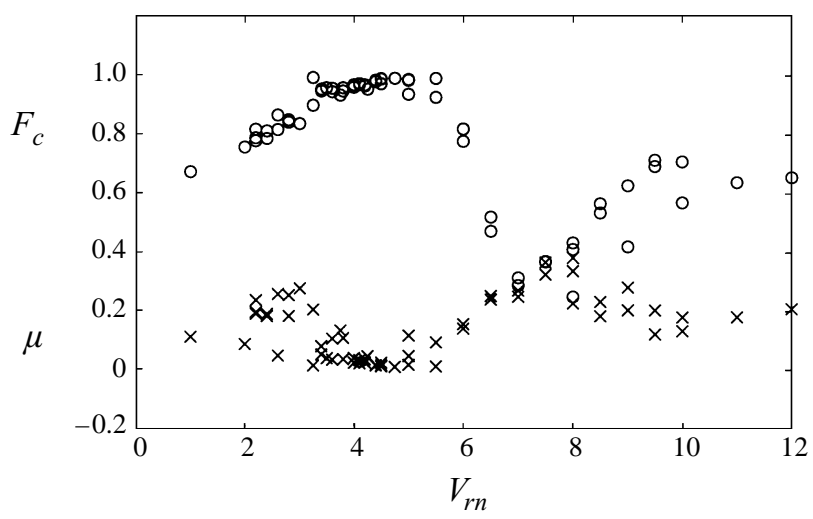

FigURE 9. Force correlations for the tapered cylinder.
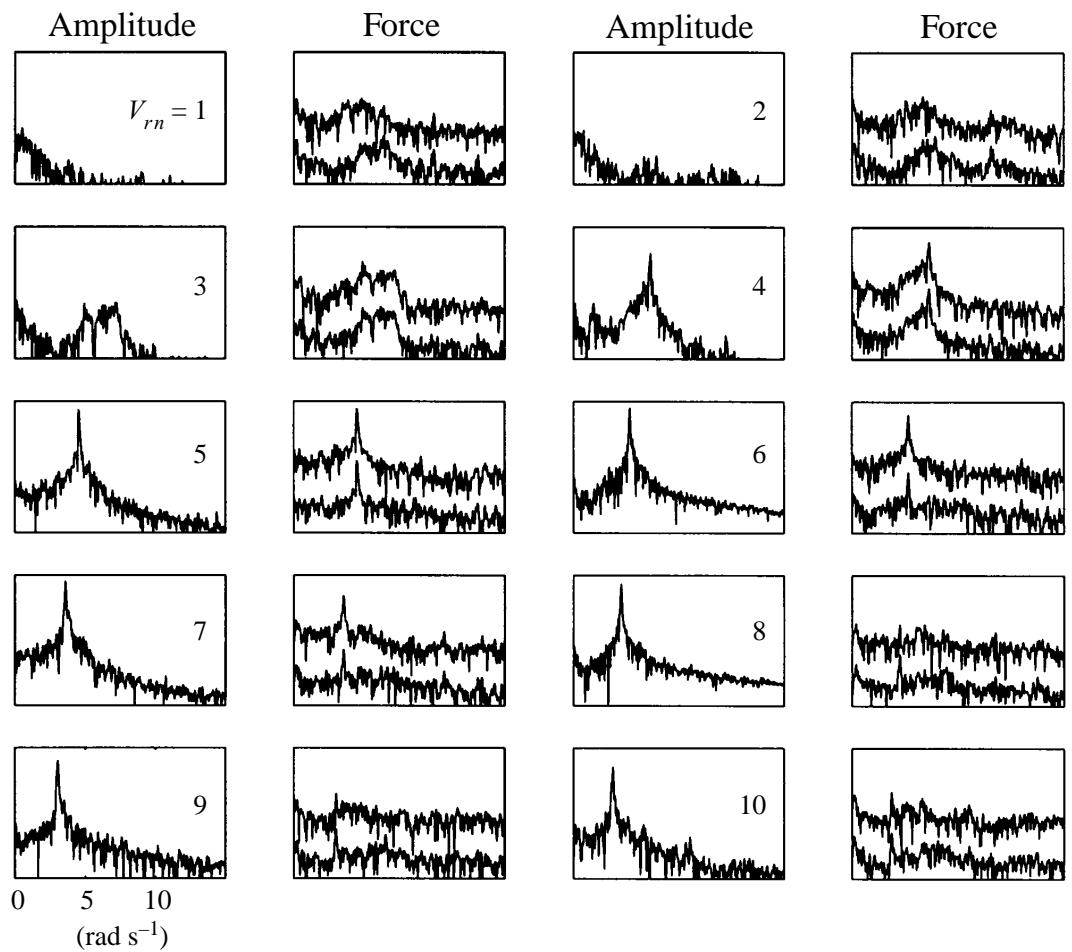

FIGURE 10. Amplitude and force power spectra for the tapered cylinder.

it is at this point that lock-in first begins, at about $90 \%$ of the structural frequency. As $V_{r n}$ increases, the observed frequency moves gradually up to a value of about 1.2, which is also the final value observed in figure 3.

Figure 10 shows displacement and force spectra for a range of reduced velocities between 1 and 10, which was also covered in the uniform cylinder case. Starting at the lower end, the force signals occupy two distinct and broad frequency ranges, consistent with two different fixed-cylinder shedding rates resulting from two diameters. The wake forces in this region are reasonably well-correlated. Some signature of the second harmonic, again broadband, emerges at $V_{r}=2.0$, while the displacement is 


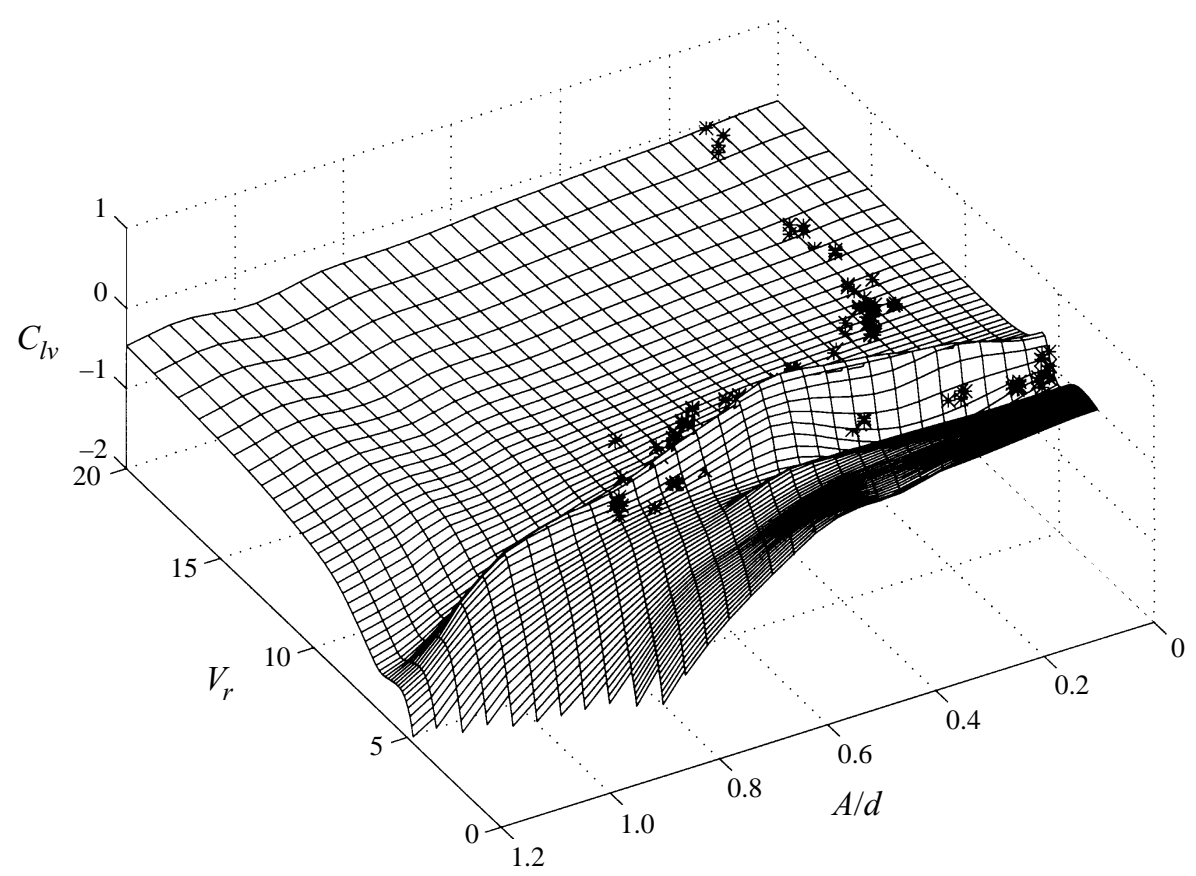

FIGURE 11. Mesh plot of $C_{l v}$ for forced oscillations, with points from free-oscillation tests (*).

largely unchanged. The spectral content of the displacement sharpens and aligns closely with that of the forces, at $V_{r n}=3.0$. At this point also, the correlation between forces increases. Both the displacement and force spectra remain remarkably narrowband for all reduced velocities between 4.0 and 7.0. Beyond this range, a broadening of the force spectra occurs; in contrast, the uniform cylinder had recovered the fixed-cylinder shedding rate in this higher range.

\section{Forced oscillations}

Forced vibration tests were conducted over the same ranges as for the free vibrations, to compare the properties of the measured lift forces between the two sets of experiments.

\subsection{Uniform cylinder}

Forced vibrations can be conducted for any combination of $\left\{V_{r}, A / d\right\}$, providing results for the complete space, whereas free vibrations depend on the mass, damping, and stiffness properties of the cylinder. In our tests, we varied only the stiffness parameter, and thus, when the free-vibration results are plotted on the $\left\{V_{r}, A / d\right\}$ space, they form a curvilinear path. Below lock-in values of $V_{r n}$, the true reduced velocity $V_{r}$ takes a roughly constant value, since the observed frequency does not vary with the structural frequency.

In figures 11-14, we show lift coefficient topographies, based on the extensive tests performed by Gopalkrishnan (1992), combined with our own current forced-vibration tests. Gopalkrishnan measured the forces at one end of the cylinder at $R e=10000$; however, we did not notice substantive deviations from our forced tests at $R e=3800$. Also shown in the figures are data points from the free-vibration tests; two lift coefficients are shown per experiment, corresponding to the forces measured at the 


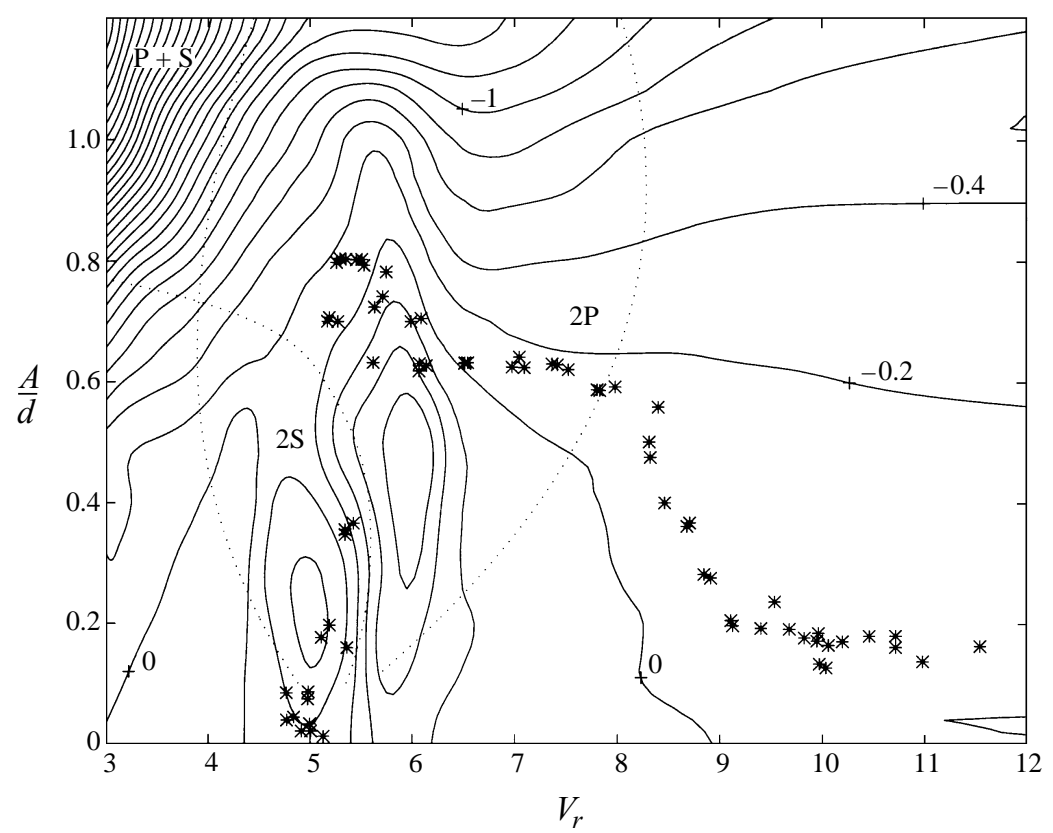

Figure 12. Contour plot of forced-oscillation $C_{l v}$ data, with an overlay of free-vibration results (*) and wake structure boundaries defined by Williamson \& Roshko (1988) $(\cdots)$.

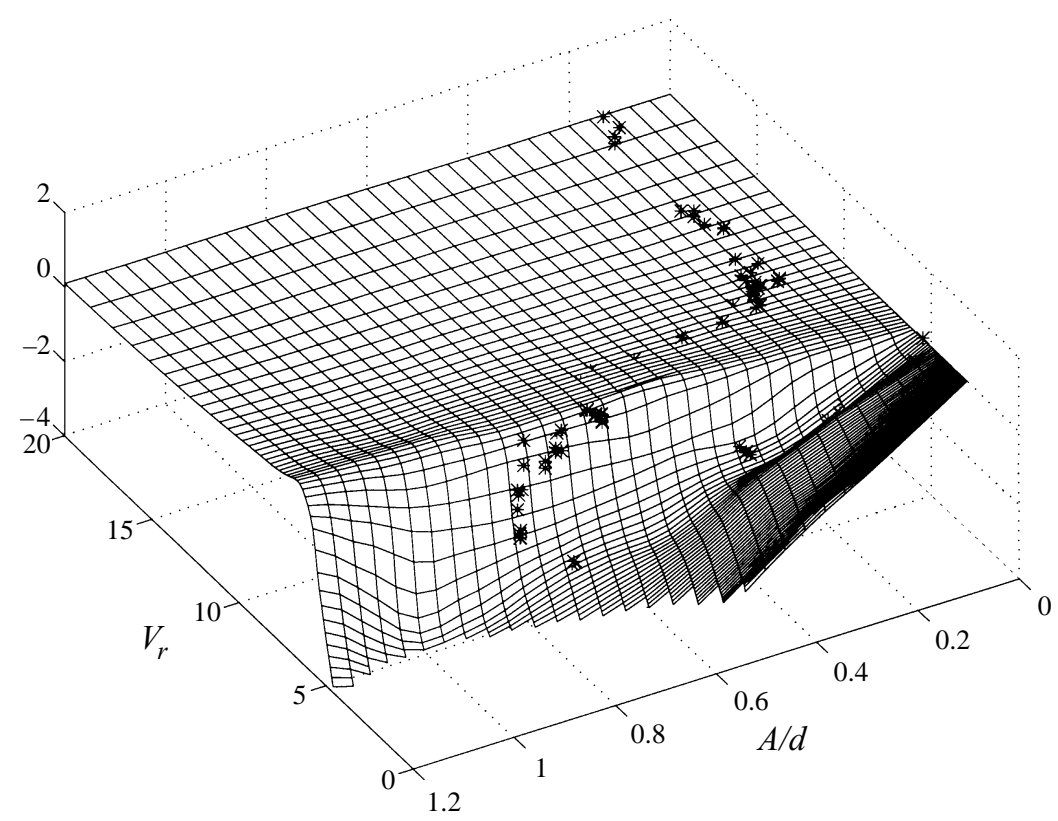

FIGURE 13. Mesh plot of $C_{l a}$ for forced oscillations, with points from free-oscillation tests (*).

two ends of the cylinder. A few of the points lie below the mesh, and so are hidden in the three-dimensional plots. Viewed on the $\left(V_{r n}, A / d\right)$-plane, the free vibrations follow the trajectory of figure 3 .

In the case of lift in phase with velocity, shown in figures 11 and 12, the free- 


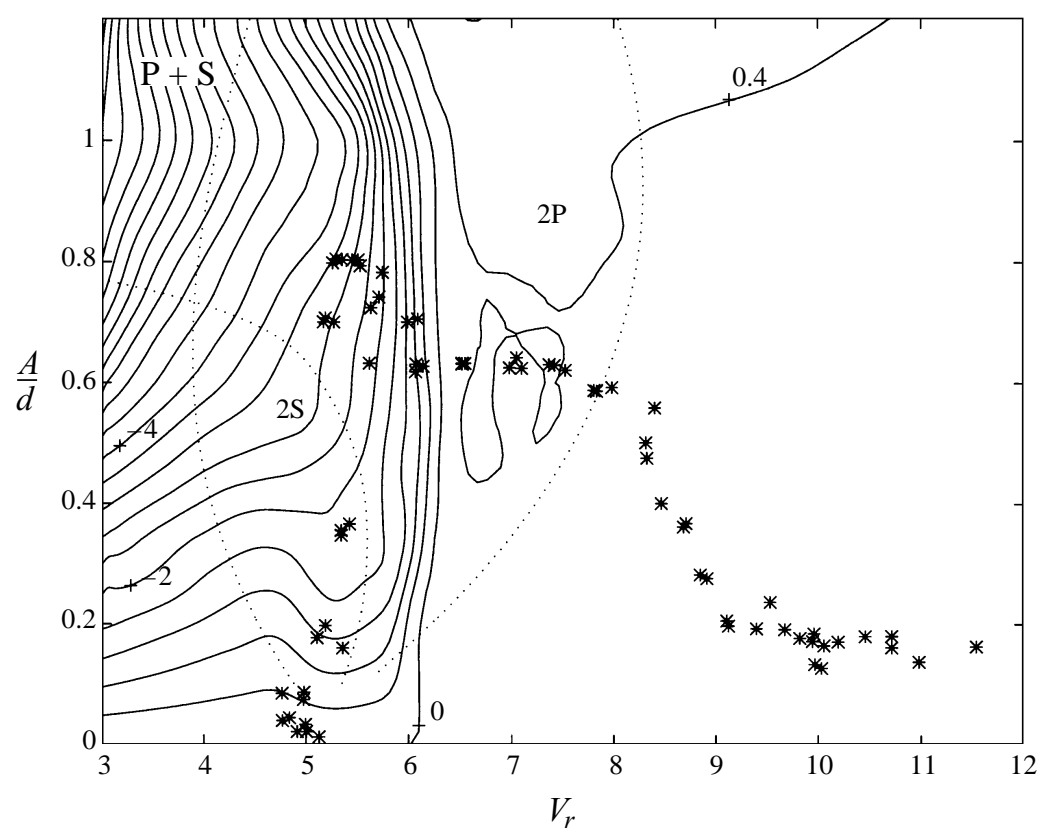

FiguRE 14. Contour plot of $C_{l a}$ for forced oscillations, with an overlay of free-vibration points (*) and wake structure boundaries defined by Williamson \& Roshko (1988) $(\cdots)$.

vibration results generally follow the zero-lift contour of the forced data, except within region III (defined in figure 4), where phase measurements are somewhat scattered. On the mesh, the low- $V_{r n}$ points traverse a fairly steep face along $V_{r} \simeq 5.5$, from amplitude zero to about $0.8 d$. The contour lines experience a sharp bend at this point, consistent with the decrease of free-vibration amplitude to $0.6 d$ for $V_{r}>6.0$. The free-vibration points settle near $A / d \simeq 0.15$ at higher reduced velocities.

Regarding the lift in phase with acceleration, the free-vibration $C_{l a}$ value becomes strongly negative as $V_{r n}$ increases, and initially follows a shallow fold (or 'valley') on the forced vibration mesh. This is shown in figures 13 and 14. The points then climb a very steep surface on the mesh, reach the crest with slightly positive value, and finally decrease to a value of zero for large $V_{r}$. Overall, the agreement between forced- and free-vibration $C_{l a}$ values is excellent.

The curves defined by Williamson \& Roshko (1988) are also shown on the contour plots of figures 12 and 14. Part of the ' $2 \mathrm{~S}$ ' to ' $2 \mathrm{P}$ ' transition line overlaps with the free-vibration data path along $V_{r} \simeq 5.5$, and is near the zero contour of $C_{l v}$, for $A / d<0.5$. This transition boundary also follows the same shallow valley in the $C_{l a}$ contour plot. Finally, the right-hand boundary of the ' $2 \mathrm{P}$ ' region passes through a 'knee' in the free-vibration amplitude response curve, at $V_{r}=8.0$ and $A / d=0.6$, as also found in Brika \& Laneville (1993).

There is good agreement between the two components of the force coefficient, $C_{l a}$ and $C_{l v}$, measured in free and forced vibrations. However a basic difference exists when the end forces are considered: the force signals have much better correlation in the forced-oscillation tests than in the free-vibration tests. Table 1 provides forcedvibration values for the force correlation coefficient, $F_{c}$, in the neighbourhood of region III. The smallest correlation coefficient for each column is shown in bold 


$\begin{array}{rcccccccc} & V_{r}: 4.5 & 4.75 & 5.0 & 5.3 & 5.55 & 5.9 & 6.3 & 6.75 \\ A / d: 0.9 & \mathbf{0 . 9 7 6} & \mathbf{0 . 9 4 4} & 0.993 & 0.992 & 0.991 & 0.990 & & \\ 0.8 & 0.993 & 0.989 & \mathbf{0 . 8 3 7} & 0.920 & 0.989 & 0.988 & 0.984 & 0.980 \\ 0.7 & 0.998 & 0.994 & 0.996 & \mathbf{0 . 8 5 6} & 0.937 & 0.975 & 0.968 & 0.955 \\ 0.6 & 0.999 & 0.999 & 0.999 & 0.998 & \mathbf{0 . 8 7 3} & 0.957 & 0.948 & 0.953 \\ 0.5 & 0.999 & 0.999 & 0.999 & 0.999 & 0.995 & 0.950 & 0.939 & 0.942 \\ 0.4 & 0.999 & 0.999 & 0.999 & 0.999 & 0.978 & \mathbf{0 . 9 1 4} & 0.951 & 0.964 \\ 0.3 & 0.998 & 0.998 & 0.998 & 0.995 & 0.997 & 0.976 & & 0.842 \\ 0.2 & 0.994 & 0.995 & 0.994 & 0.994 & 0.927 & 0.969 & & 0.806\end{array}$

TABLE 1. Forced-oscillation values of $F_{c}$
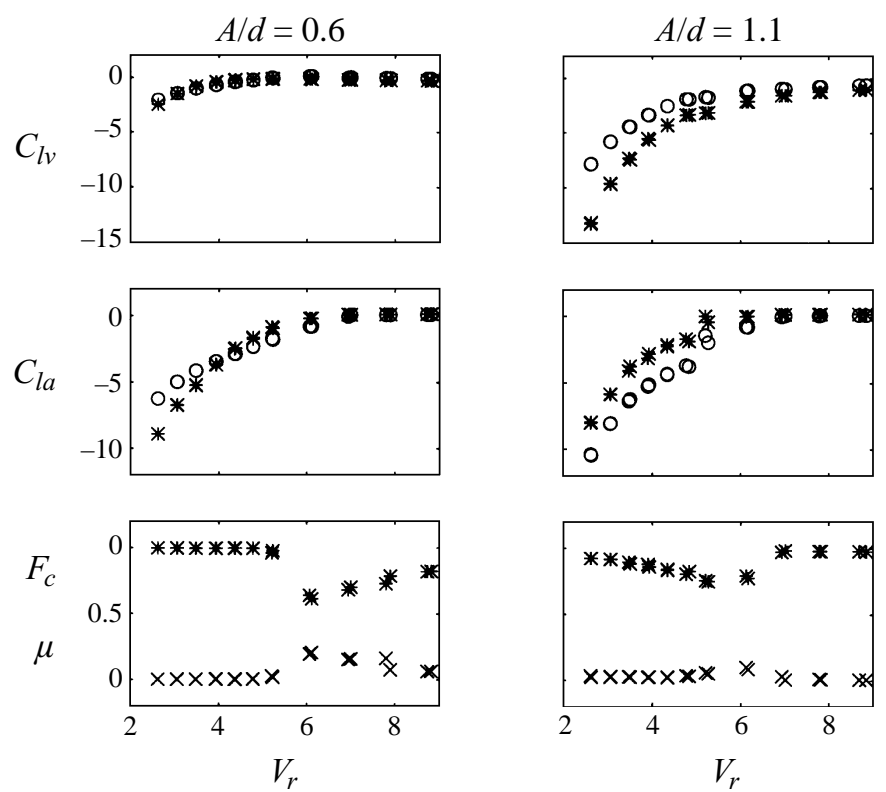

FIGURE 15. Forced-oscillation tests with a 40:1 tapered cylinder. For $C_{l v}$ and $C_{l a}$, denotes the narrow end of the cylinder, $\circ$ denotes the wide end.

lettering: the lowest coefficients are in the range of 0.84 to 0.87 , always much higher than the lowest free-vibration coefficients, which were in the range $0-0.5$.

\subsection{0:1 tapered cylinder}

We performed a set of forced vibrations with the 40:1 tapered cylinder to explore the mapping of forces between free and forced vibrations. Figure 15 provides a summary of the measured forces for two $A / d$ values of 0.6 and 1.1, and a range of reduced velocity $V_{r}$. For each amplitude three plots are shown, providing $(a)$ the lift coefficients (at the two ends of the cylinder section) in phase with velocity, $C_{l v} ;(b)$ the lift coefficients in phase with acceleration, $C_{l a}$; and $(c)$ the force cross-correlation coefficient $F_{c}$ and standard deviation $\mu$. For both amplitudes, both components of the lift coefficients are strongly negative at low $V_{r}$, but increase to near zero above $V_{r}=7.0$. In contrast with the uniform-cylinder results, there were no tests yielding positive lift coefficients.

Although there are still differences, the force correlation coefficient for the tapered 
cylinder performing forced oscillations matches the free-vibration results better than the uniform cylinder. This is especially true at $A / d=0.6$, near the maximum amplitude of the free-vibrations. A comparison of figure 15 with figure 9 shows the closer similarity of the two sets of experiments. Transition occurs at the same reduced velocity $(\simeq 5.75)$; the primary difference is the minimum value for the correlation coefficient $F_{c}$, which is equal to 0.28 for the free-vibration tests, and 0.58 for the forced-vibration results.

As in the case of the uniform cylinder, in forced-oscillation tests larger imposed amplitudes tend to correlate the forces better.

\section{Discussion}

The use of a force-feedback closed-loop control system to effect an equivalent system with desired properties solves several of the major problems associated with free-vibration tests.

- The apparatus allows for low-mass tests; the only real limit is the mass of the test cylinder, which dictates the magnitude of the inertial correction. This inertial correction is inherently destabilizing, so the critical constraint is that $M-\bar{M}>0$.

- Force and displacement measurements involve various fittings and wiring. By using a servo-controlled approach, the effects of these connections on the dynamic response of the system can be eliminated. One limitation of the system is the small phase loss incurred by filtering the force signals. However, the data, which match previously reported results closely, suggest that this has only minor effects.

- The apparatus allows for conducting both forced- and free-vibration testing under identical conditions, since only a software change is required. The nature of the feedback system, as noted previously, can also provide multi-mode and nonlinear structural models: preliminary work with multi-mode models has been reported by Hover et al. (1997).

Recording the two cylinder end forces separately allows for a study of distributed force correlation and hence, indirectly, flow-correlation along the section used. The loss of force correlation of free vibrations in region III, lying between region II, associated with ' $2 \mathrm{~S}$ ' shedding, and region IV, associated with ' $2 \mathrm{P}$ ' shedding, has a plausible explanation. In region III, repeated switching between upper and lower branches of the amplitude hysteresis loop may occur, accompanied by non-uniform flow conditions along the span of the cylinder. Hence, whereas there is no discontinuity in the amplitude of the lift coefficient during ' $2 \mathrm{~S}$ ' to ' $2 \mathrm{P}$ ' transition, there is phase transition, causing loss of correlation. This view is reinforced by the results in Techet, Hover \& Triantafyllou (1998) which show flow visualization at Reynolds numbers in the range 400 to 1500 , behind a 40:1 tapered rigid cylinder. It is shown that under certain conditions a hybrid mode forms, consisting of a ' $2 S$ ' pattern along part of the cylinder span having the larger diameter, connected through a vortex split to a '2P' pattern along the small diameter part. The pattern is repeatable within one cylinder diameter from cycle to cycle. Our results herein, reporting loss of correlation for parametric ranges close to those in Techet et al. (1998), lead to the tentative explanation that a spanwise ' $2 \mathrm{~S}$ ' to ' $2 \mathrm{P}$ ' transition is the cause of force correlation loss. This effect may be stronger in free vibrations of uniform cylinders, when small variations in frequency and amplitude of oscillation can cause the vortex split to shift location more dramatically along the span.

The correspondence between $C_{l a}$ in forced- and free-vibration tests is remarkably close, and indicates that the added mass component of VIV can be parametrized 
successfully from forced experiments. Similarly, zero power flow in the forced oscillations holds on a contour which is fairly close to the free-vibration trajectory, noteworthy because forced vibrations cannot enforce zero power flow. Beyond these similarities, however, forced vibrations seem to exert greater control on the mode transition, resulting in a smaller loss of correlation: the forced vibrations preserve correlation of the two end forces better than the free vibrations. In the free-vibration tests, some runs in region III approach zero correlation, but the lowest value in table 1 is 0.837 . Additionally, the path of minimum correlation, obtained from table 1 , follows a narrow valley on the $\left(A / d, V_{r}\right)$ plane, which does not coincide with the path of the free-vibration trajectory (see, for example, figure 12). This further differentiates the free- from the forced-vibration results.

\section{Conclusions}

A novel force-feedback control system was used to explore free and forced vibrations of uniform and tapered rigid cylinder sections, providing measurements of the forces at both ends of the section. Cross-correlation between the two end forces shows distinct regions of high- and low-correlation; a region of low force correlation (region III in figure 4) is found around the Strouhal frequency.

Free-vibration tests of a uniform cylinder with low equivalent structural damping yield the amplitude response as a function of nominal reduced velocity, in agreement with previous results (Khalak \& Williamson 1996). In region III, for the range of reduced velocities between 5.0 and 6.25 , correlation coefficients as low as zero are observed, coincident with abrupt phase changes between tests. Comparison with forced vibration data shows good agreement of the lift force coefficients in phase with velocity $C_{l v}$ and in phase with acceleration $C_{l a}$. The correlation coefficient for forced vibrations in region III, however, drops only to a value of 0.83 , showing better correlated force (and flow) conditions along the span.

Free- and forced-vibration tests of tapered rigid cylinders with 40:1 taper also show good agreement in the values of the lift coefficients $C_{l v}$ and $C_{l a}$, while the force correlation values agree better than in the case of the uniform cylinder. Differences between the uniform and tapered cylinder free-vibration results are also noted, including an expanded region of low correlation around the Strouhal frequency, with a more gradual transition from good to poor correlation. This gradual variation applies to other parameters as well, such as phase, in contrast with the uniform cylinder results. Furthermore, the tapered cylinder free vibrations allow lock in to occur at lower values of $V_{r n}$ than for the uniform cylinder.

We would like to acknowledge financial support from the Office of Naval Research (Ocean Engineering Division), under contract N00014-95-1-0106 and monitored by Dr T. F. Swean Jr.

\section{REFERENCES}

Bishop, R. E. D. \& Hassan, A. Y. 1964 The lift and drag forces on a circular cylinder oscillating in a flowing fluid. Proc. R. Soc. Lond. A 277, 51-75.

Blevins, R. 1990 Flow-Induced Vibration. Van Nostrand Reinhold.

BriKa, D. \& LANEville, A. 1993 Vortex-induced vibrations of a long flexible circular cylinder. J. Fluid Mech. 250, 481-508.

Coutanceau, M. \& Defaye, J. R. 1991 Circular cylinder wake configurations: A flow visualization survey. Appl. Mech. Rev. 44, 255-305. 
FENG, C. C. 1968 The measurement of vortex-induced effects in flow past stationary and oscillating circular and D-section cylinders. MASc Thesis, University of British Columbia.

Gopalkrishnan, R. 1992 Vortex-induced forces on oscillating bluff cylinders. PhD Dissertation, Massachusetts Institute of Technology/Woods Hole Oceanographic Institution Joint Program in Oceanographic Engineering.

Gopalkrishnan, R., Grosenbaugh, M. A. \& Triantafyllou, M. S. 1992 Amplitude modulated cylinders in constant flow: Fundamental experiments to predict response in shear flow. $P V P$ 245, Bluff-Body/Fluid and Hydraulic Machine Interactions (ed. M. P. Paidoussis, C. Dalton \& D. S. Weaver). Book G00727, ASME.

GrIFFIN, O. E. 1972 Flow near self-excited and forced vibrating circular cylinders. J. Engng Industry May 1972, 539-547.

Hover, F. S., Miller, S. N. \& Triantafyllou, M. S. 1997 Vortex-induced vibration of marine cables: Experiments using force feedback. J. Fluids Struct. 11, 306-326.

Humphries, J. A. 1987 Vortex induced vibrations in sheared flow. PhD Dissertation, Cranfield Institute of Technology.

KhalaK, A. \& Williamson, C. H. K. 1996 Dynamics of a hydroelastic cylinder with very low mass and damping. J. Fluids Struct. 10, 455-472.

NAKANO, M. \& Rockwell, D. 1994 Flow structure in the frequency-modulated wake of a cylinder. J. Fluid Mech. 266, 93-119.

Naudascher, E. \& Rockwell, D. 1994 Flow-Induced Vibrations. A.A. Balkema.

Newman, D. \& Karniadakis, G. E. 1996 Simulations of flow over a flexible cable: A comparison of forced and flow-induced vibration. J. Fluids Struct. 10, 439-453.

Parkinson, G. 1989 Phenomena and modelling of flow-induced vibrations of bluff bodies. Prog. Aerospace Sci. 26, 169-224.

SARPKAYA, T. 1977 Transverse oscillations of a circular cylinder in uniform flow. Naval Postgraduate School, Monterey California: Rep. NPS-69SL77071.

SARPKAYA, T. 1995 Hydrodynamic damping, flow-induced oscillations, and biharmonic response. J. Offshore Mech. Arctic Engng 117, 232-238.

StAuBLI, T. 1983 Calculation of the vibration of an elastically mounted cylinder using experimental data from forced oscillation. Trans. ASME: J. Fluids Engng 105, 225-229.

Techet, A. H., Hover, F. S. \& Triantafyllou, M. S. 1998 Vortical patterns behind tapered cylinders oscillating transversely to a uniform flow. J. Fluid Mech. 363, 79-96.

Williamson, C. H. K. 1996 Vortex dynamics in the wake. Ann. Rev. Fluid Mech. 28, 477-539.

Williamson, C. H. K. \& Roshko, A. 1988 Vortex formation in the wake of an oscillating cylinder. J. Fluids Struct. 2, 355-381. 